\title{
The Global Markov Property for Equilibrium States Which are Determined by Correlations in a Strip
}

\author{
R. Kuik \\ Institute for Theoretical Physics, Postbox 800, NL-9700 AV Groningen, The Netherlands
}

\begin{abstract}
Consider an interaction-round-face potential on the lattice $\mathbb{Z}^{d}$, which may include nearest-neighbor and next-nearest-neighbor pair interactions, as well as more general plaquette terms. Assuming some periodicity of the potential it is shown that, under the condition that equilibrium states can be distinguished by expectation values of sufficiently local observables, equilibrium states possess a global Markov property. The condition under which this Markov property is shown to hold is met in particular in case the equilibrium state is unique or determined by the magnetization. The proof is based on an application of the variational principle to states which are constructed like Markov chains.
\end{abstract}

\section{Introduction}

We consider systems of classical spins on $\mathbb{Z}^{d}$ with, per lattice point, values in a compact metric space. The Markov property entails that upon fixing the configuration on the boundary of a volume the distribution of the spin configurations inside the boundary becomes independent of the spin configuration outside the boundary. The very definition of Gibbs states for a finite range potential states that Markov properties hold for finite volumes, see e.g. [1]. In this case one speaks of a local Markov property. In case such a property holds for an infinite volume this is called a global Markov property.

Gibbs states for finite-range potentials possess thus local Markov properties. However, the question whether or not Gibbs states possess global Markov properties is not easy to answer. Indeed, for the three-dimensional Ising model Goldstein has constructed an example of a Gibbs state that does not have the global Markov property with respect to a plane, [1]. Other examples of this type are given in [10].

Global Markov properties are an important ingredient in Nelson's scheme of Euclidean field theory because these properties are essential in constructing the Hamiltonian of the theory (see e.g. [2]). Despite their importance, global Markov properties are known to hold in relatively few cases. These cases can be divided into two main groups: 
A) The interaction is attractive or such that F.K.G. inequalities hold. The maximal Gibbs measure ( + magnetization) and the minimal Gibbs measure ( - magnetization) then have global Markov properties. Such cases are discussed in $[1,3$, and 4$]$.

B) The potential satisfies Dobrushin's uniqueness criterion, implying that there exists a unique Gibbs state, cf. [9]. This unique Gibbs state then has the global Markov property with respect to all surfaces, [3 and 5].

One consequence of Theorem 1 below is that these results can be extended: If the interaction has the properties specified in Theorem 1 and admits a unique Gibbs state then this state has a global Markov property specified below.

This paper is inspired by methods used by Schlijper in [6]. In particular the technique of entropy inequalities used in Lemma 2 is taken from [6].

The proof of the main result (Theorem 1) of this paper uses the variational principle. Briefly outlined the argument goes as follows. Consider a state on two planes of the lattice. A construction is presented which yields a state on all of the lattice having a global Markov property and which is an extension of the state on the two planes. [In fact two versions of such a construction are presented in this paper.] This construction is then applied to the restriction $\mu_{2}$, to two planes of an equilibrium state $\mu$ on $\mathbb{Z}^{d}$ yielding a (new) state $\bar{\mu}$ on all of $\mathbb{Z}^{d}$. If $\mu$ already had the global Markov property then $\mu=\bar{\mu}$, but irrespective of this it is shown that $\bar{\mu}$ always is an equilibrium state (with a global Markov property and coinciding with $\mu$ on the two planes).

The proof that $\bar{\mu}$ is an equilibrium state rests on two observations,

1) the energy density of $\mu$ equals the energy density of $\bar{\mu}$,

2) the entropy density of $\bar{\mu}$ is not smaller than the entropy density of $\mu$, since these two observations, by the variational principle (Theorem 0 ), imply that $\bar{\mu}$ is also an equilibrium state. The second of the just mentioned observations is obtained making use of two lemmas (Lemma 1 and Lemma 2). Theorem 2, for the case of a two-dimensional lattice, states a further relation between the constructed state $\bar{\mu}$ and the original equilibrium state $\mu$.

We introduce some notation. Let $\Omega_{0}$ be a compact metric space. For $\Lambda \subseteq \mathbb{Z}^{d}$ we put $\Omega_{\Lambda}=\left(\Omega_{0}\right)^{\Lambda}$. We write $\Omega_{\mathbb{Z}^{d}}=\Omega$. We equip $\Omega_{\Lambda}$ with the product topology and denote by $C\left(\Omega_{\Lambda}\right)$ the space of continuous functions on $\Omega_{\Lambda}$. Let $\alpha_{\Lambda, \Lambda^{\prime}}: \Omega_{\Lambda^{\prime}} \rightarrow \Omega_{\Lambda}$ be the restriction map for $\Lambda^{\prime} \supset \Lambda$. We write $\alpha_{\Lambda}=\alpha_{\Lambda, \mathbb{Z}^{d}}$. Define $\mathfrak{F}_{\Lambda}=\{f: \Omega \rightarrow \mathbb{C} \mid$; there exists $f_{\Lambda}: \Omega_{\Lambda} \rightarrow \mathbb{C}$ such that $\left.f=f_{\Lambda} \cdot \alpha_{\Lambda}\right\}$. Denote by $\mathfrak{F}(\Lambda)$ the set of complex valued functions on $\Omega_{\Lambda}$.

The map

$$
\mathfrak{F}(\Lambda) \ni f_{\Lambda} \rightarrow f_{\Lambda} \alpha_{\Lambda} \in \mathfrak{F}_{\Lambda}
$$

gives then a one-to-one correspondence between $\mathfrak{F}(\Lambda)$ and $\mathfrak{F}_{\Lambda}$. Henceforth we will identify $\mathfrak{F}(\Lambda)$ and $\mathfrak{F}_{\Lambda}$ by this one-to-one correspondence. Under this correspondence

$$
C\left(\Omega_{\Lambda}\right)=C_{\Lambda} \equiv\left\{f \in C(\Omega) \mid \text { there exists } f_{\Lambda} \in C\left(\Omega_{\Lambda}\right) \text { such that } f=f_{\Lambda} \cdot \alpha_{\Lambda}\right\} .
$$

Denote by $\mathfrak{B}_{\Lambda}$ the smallest $\sigma$-algebra of subsets of $\Omega$ with the property that functions from $C_{\Lambda}$ are measurable with respect to it. $\mathfrak{B}_{\Lambda}$ is the $\sigma$-algebra generated by cylinder sets with finite base in $\Lambda$. Write $\mathfrak{B}_{\mathbb{Z}^{d}}=\mathfrak{B}$. Let $\mathfrak{B}(\Lambda)$ be the Borel $\sigma$-algebra 
of $\Omega_{\Lambda}$. Upon identifying sets with their characteristic function, $\mathfrak{B}(\Lambda)$ as a subset of $\mathfrak{F}(\Lambda)$ is identified with $\mathfrak{B}_{\Lambda} \subset \mathfrak{F}_{\Lambda}$. States on $C_{\Lambda}=C\left(\Omega_{\Lambda}\right)$ are identified with probability measures on $\mathfrak{B}_{\Lambda}=\mathfrak{B}(\Lambda)$, through the Riesz-Markov Theorem.

For $k \in \mathbb{Z}$ we introduce $P_{k}=\left\{x \in \mathbb{Z}^{d} \mid x_{1}=k\right\}$ and

$$
\left(\mathbb{Z}^{d}\right)_{k, \delta}=\left\{x \in \mathbb{Z}^{d} \mid \delta x_{1} \geqq 0\right\} \quad \text { for } \quad \delta= \pm .
$$

We say that a state $\mu$ on $C(\Omega)$ has the Markov property with respect to $P_{k^{\prime}}$ if $\mathbb{E}_{k}^{\mu} f=$ $\mathbb{E}_{\leqq k}^{\mu} f$ for all $f \in L^{2}\left(\mu ; \mathfrak{B}_{\left(\mathbb{Z}^{d}\right)_{k,+}}\right)$, where $\mathbb{E}_{k}^{\mu}$ and $\mathbb{E}_{\leqq k}^{\mu}$ are orthogonal projections on $L^{2}(\mu ; \mathfrak{B})$ with ranges given respectively by $L^{2}\left(\mu ; \mathfrak{B}_{P_{k}}\right)$ and $L^{2}\left(\mu ; \mathfrak{B}_{\left(\mathbb{Z}^{d}\right)_{k,-}}\right)$.

For $a \in \mathbb{Z}^{d}$ consider $\tau^{(a)}: \mathbb{Z}^{d} \rightarrow \mathbb{Z}^{d}$ which maps $x \in \mathbb{Z}^{d}$ onto $\tau^{(a)} x \in \mathbb{Z}^{d}$ given by $\tau^{(a)} x=x+a$. By transpositions (with the inverse) $\mathbb{Z}^{d}$ acts on $\Omega$, and $\mathfrak{F}_{\mathbb{Z}^{d}}$. Note that $C(\Omega)$ is invariant under this action. Thus by one more transposition $\mathbb{Z}^{d}$ acts on $E_{C(\Omega)}$, where $E_{C(\Omega)}$ is the set of states on $C(\Omega)$. All these actions of $a \in \mathbb{Z}^{d}$ on the various spaces are also denoted by $\tau^{(a)}$. Then, for instance, $\tau^{(a)} \mathfrak{F}_{\Lambda}=\mathfrak{F}_{\Lambda+a}$.

$$
\text { Let } \Phi: \bigcup_{\substack{\Lambda \subset \mathbb{Z}^{d} \\ \Lambda \text { finite }}} \Omega_{\Lambda} \rightarrow \mathbb{R},
$$

with $\Phi \circ \alpha_{\Lambda} \in C\left(\Omega_{\Lambda}\right)$ for all $\Lambda$ finite, be a potential which is nonzero only round faces of $\mathbb{Z}^{d}$, i.e., $\Phi \circ \alpha_{X} \equiv 0$ whenever there exist $x, y \in X$ such that maximum $\left\{\mid x_{i}-\right.$ $\left.y_{i}|| i=1, \ldots, d\right\}>1$.

We will make use of the variational principle. To this end we assume that $\Phi$ is $H$-invariant, i.e., $\Phi \circ \tau^{(a)}=\Phi$ for all $a \in H$, where $H \subseteq \mathbb{Z}^{d}$ is a subgroup of finite index in $\mathbb{Z}^{d}$. Let $\mu_{0}$ be a probability measure on $\Omega_{0}$. Denote by $\mu_{0}^{(\Lambda)}$ the product measure on $\Omega_{\Lambda}=\left(\Omega_{0}\right)^{\Lambda}$ constructed from $\mu_{0}$.

The pressure $P_{\Phi}$ is defined by

$$
P_{\Phi}=\lim _{\Lambda \uparrow \mathbb{Z}^{d}} \frac{1}{|\Lambda|} \ln Z_{\Lambda},
$$

where

$$
Z_{\Lambda}=\int_{\Omega_{\Lambda}} \mu_{0}^{(\Lambda)}\left(d \xi_{\Lambda}\right) \exp \left[-H_{\Lambda}\left(\xi_{\Lambda}\right)\right]
$$

with

$$
H_{\Lambda}\left(\xi_{\Lambda}\right)=\sum_{X \subseteq \Lambda} \Phi \circ \alpha_{X, \Lambda}\left(\xi_{\Lambda}\right)
$$

Lim denotes limit in the sense of van Hove and $|\Lambda|$ denotes the number of points $\Lambda \uparrow \mathbb{Z}^{d}$ of $\Lambda \subset \mathbb{Z}^{d}$.

If $\mu$ is a state on $C(\Omega)$ such that its restriction to $C\left(\Omega_{\Lambda}\right)$ is absolutely continuous with respect to $\mu_{0}^{(\Lambda)}$ we denote its Radon-Nikodym derivative by $\mu^{(\Lambda)}$. The average entropy density per lattice point, $s(\mu)$, of a $G$-invariant state $\mu$ where $G$ is a subgroup of $\mathbb{Z}^{d}$ with finite index in $\mathbb{Z}^{d}$, is defined by

$$
s(\mu)=\lim _{\Lambda \uparrow \mathbb{Z}^{d}} \frac{1}{|\Lambda|} S_{\Lambda}(\mu)
$$


with $S_{\Lambda}(\mu)=-\int_{\Omega_{\Lambda}} \mu_{0}^{(\Lambda)}\left(d \xi_{\Lambda}\right)\left(\mu^{(\Lambda)}\left(\xi_{\Lambda}\right) \ln \mu^{(\Lambda)}\left(\xi_{\Lambda}\right)\right)$ if the restriction of $\mu$ to $C\left(\Omega_{\Lambda}\right)$ is absolutely continuous with respect to $\mu_{0}^{(\Lambda)}$ and $S_{\Lambda}(\mu)=-\infty$ otherwise.

Let $M_{0} \subset \mathbb{Z}^{d}$ be such that $\left\{\tau^{(a)} M_{0}\right\}_{a \in G}$ is a partition of $\mathbb{Z}^{d}$. Note that $\left|M_{0}\right|<\infty$ since $G$ has finite index in $\mathbb{Z}^{d}$. Suppose $\left\{\Lambda_{n}\right\}_{n \in \mathbb{N}}$ is a van Hove sequence such that each $\Lambda_{n}$ is a union of sets from the collection $\left\{\tau^{(a)} M_{0}\right\}_{a \in G}$. Then $s(\mu)=\inf _{n \in \mathbb{N}}\left(1 /\left|\Lambda_{n}\right|\right) S_{\Lambda_{n}}(\mu)$. In particular $s(\mu) \leqq\left(1 /\left|\Lambda_{n}\right|\right) S_{\Lambda_{n}}(\mu)$ for all $n \in \mathbb{N}$. Furthermore the functional $s$ is upper-semicontinuous on the set of $G$-invariant states.

The proofs of these assertions for a general subgroup $G \subseteq \mathbb{Z}^{d}$ with finite index are a modification of the proof in case $G=\mathbb{Z}^{d}$, cf. [8]. For $x \in \mathbb{Z}^{d}$ define $A_{\Phi}^{(x)} \in C(\Omega)$ by

$$
A_{\Phi}^{(x)}=\sum_{X}^{(x)} \Phi \circ \alpha_{X}
$$

where $\sum_{X}^{(x)}$ means taking the sum over finite subsets of $X$ of $\mathbb{Z}^{d}$ with $x$ as first element of $X$ for the lexicographic order of $\mathbb{Z}^{d}$.

The variational principle is stated in the next theorem where $E_{C(\Omega)}^{G} \subset E_{C(\Omega)}$ is the set of $G$-invariant states and where $\mathscr{G}_{\Phi}$ is the set of Gibbs states for the potential $\Phi$.

\section{Theorem 0.}

1. For all $\mu \in E_{C(\Omega)}^{G}, s(\mu)-\frac{1}{\left|M_{0}\right|} \sum_{x \in M_{0}} \mu\left(A_{\Phi}^{(x)}\right) \leqq P_{\Phi}$.

2. If $\mu \in E_{C(\Omega)}^{G}$, then

$$
\mu \in \mathscr{G}_{\Phi} \Leftrightarrow s(\mu)-\frac{1}{\left|M_{0}\right|} \sum_{x \in M_{0}} \mu\left(A_{\Phi}^{(x)}\right)=P_{\Phi} .
$$

\section{The Main Result}

Introduce $P_{k}(N)=\left\{x \in \mathbb{Z}^{d} \mid x_{1}=k ;-N \leqq x_{i}<N\right.$ for $\left.i=2, \ldots, d\right\}$, where $k \in \mathbb{Z}$ and $N \in \mathbb{N} \cup\{\infty\}$. Let $\mu_{2}^{(N)}$ be a state on $C_{P_{0}(N) \cup P_{1}(N)}$ and let $\mathbb{E}_{\downarrow}^{(N)}$ and $\mathbb{E}_{\uparrow}^{(N)}$ be the orthogonal projections in $L^{2}\left(\mu_{2}^{(N)} ; \mathfrak{B}_{P_{0}(N) \cup P_{1}(N)}\right)$ with ranges given respectively by $L^{2}\left(\mu_{2}^{(N)} ; \mathfrak{B}_{P_{0}(N)}\right)$ and $L^{2}\left(\mu_{2}^{(N)} ; \mathfrak{B}_{P_{1}(N)}\right)$. Put $\bar{\Lambda}^{(N)}=\left\{x \in \mathbb{Z}^{d} \mid-N \leqq x_{i}<N\right.$ for $\left.i=2, \ldots, d\right\}$ and put $\tau_{1}=\tau^{((1,0, \ldots, 0))}$ for $(1,0, \ldots, 0) \in \mathbb{Z}^{d}$.

Next we introduce two states $\bar{\mu}_{\text {alt }}^{(N)}$ and $\bar{\mu}_{\text {inv }}^{(N)}$ which are extensions of $\mu_{2}^{(N)}$ to $C_{\bar{\Lambda}^{(N)} \text {. }}$ The state $\bar{\mu}_{\text {alt }}^{(N)}$ will be constructed such that it is reflectionally invariant. In fact $\bar{\mu}_{\mathrm{alt}}^{(N)}$ is the unique state on $C_{\bar{\Lambda}^{(N)}}$ that is a reflectionally invariant extension of $\mu_{2}^{(N)}$ which has the Markov property with respect to $P_{0}(N)$ and $P_{1}(N)$. Formally, let $\bar{\mu}_{\text {alt }}^{(N)}$ be the state on $C_{\bar{\Lambda}^{(N)}}$ defined by

$$
\begin{aligned}
\bar{\mu}_{\mathrm{alt}}^{(N)}\left(\prod_{k=-L}^{L} f_{l}\right)= & \int d \mu_{2}^{(N)}\left\{\left[\mathbb{E}_{\downarrow}^{(N)}\left(\tilde{f}_{-1} \mathbb{E}_{\uparrow}^{(N)}\left(\tilde{f}_{-2} \cdots \mathbb{E}_{(-1)^{-L_{\uparrow}}}\left(\tilde{f}_{-L}\right) \cdots\right)\right)\right]\right. \\
& \left.\cdot f_{0} f_{1}\left[\mathbb{E}_{\uparrow}^{(N)}\left(\tilde{f}_{2} \mathbb{E}_{\downarrow}^{(N)}\left(\tilde{f}_{3} \cdots \mathbb{E}_{(-1)^{L}}\left(\tilde{f}_{L}\right) \cdots\right)\right)\right]\right\}
\end{aligned}
$$

for $L \in \mathbb{N}$ and $f_{l} \in C_{P_{l}(N)} ; l=-L, \ldots, L$. 
We have introduced $\tilde{f}_{l}=\tau_{1}^{-l+\delta_{-1,(-1)^{l}}} f_{l}$ and also $-1 \uparrow=\downarrow$ and $+1 \uparrow=\uparrow$. In case $\mu_{2}^{(N)}$ satisfies $\mu_{2}^{(N)}\left(\tau_{1} f\right)=\mu_{2}^{(N)}(f)$ for all $f \in C_{P_{0}(N)}$ we also introduce the state $\bar{\mu}_{\text {inv }}^{(N)}$ on $C_{\bar{\Lambda}^{(N)}}$. The state $\bar{\mu}_{\text {inv }}^{(N)}$ is the unique state on $C_{\bar{A}^{(N)}}$ that is a $\tau_{1}$-invariant extension of $\mu_{2}^{(N)}$ to $C_{\bar{\Lambda}^{(N)}}$ which has the Markov property with respect to $P_{0}(N)$ and is defined by

$$
\begin{aligned}
\bar{\mu}_{\text {inv }}^{(N)}\left(\prod_{l=-L}^{L} f_{l}\right)= & \int d \mu_{2}^{(N)}\left\{\left[\tau_{1}^{-1} \mathbb{E}_{\uparrow}^{(N)}\left(f_{-1}^{\prime} \tau_{1}^{-1} \mathbb{E}_{\uparrow}^{(N)}\left(f_{-2}^{\prime} \cdots \tau_{1}^{-1} \mathbb{E}_{\uparrow}^{(N)}\left(f_{-L}^{\prime}\right) \cdots\right)\right)\right]\right. \\
& \left.\cdot f_{0} f_{1}\left[\tau_{1} \mathbb{E}_{\downarrow}^{(N)}\left(f_{2}^{\prime} \tau_{1} \mathbb{E}_{\downarrow}^{(N)}\left(f_{3}^{\prime} \cdots \tau_{1} \mathbb{E}_{\downarrow}^{(N)}\left(f_{L}^{\prime}\right) \cdots\right)\right)\right]\right\}
\end{aligned}
$$

for $L \in \mathbb{N}$ and $f_{l} \in C_{P_{l}(N)} ; l=-L, \ldots, L$. We have introduced

$$
f_{l}^{\prime}=\left\{\begin{array}{lll}
\tau_{1}^{-l} f_{l} & \text { when } & l \leqq 0 \\
\tau_{1}^{-l+1} f_{l} & \text { when } & l>0
\end{array}\right.
$$

The method given above for constructing $\bar{\mu}_{\text {alt }}^{(N)}$ and $\bar{\mu}_{\text {inv }}^{(N)}$ relates to a method discussed in [2], see pages 122-125 and references given there. Let $\theta_{k}$ be the reflection of the lattice in the plane $P_{k}$. Thus $\theta_{k}$ maps $x \in \mathbb{Z}^{d}$ onto $\theta_{k} x \in \mathbb{Z}^{d}$, where $\theta_{k} x$ is given by $\left(\theta_{k} x\right)_{1}=2 k-x_{1}$ and $\left(\theta_{k} x\right)_{i}=x_{i}$ for $i=2, \ldots, d$. A reflection $\theta_{k}$ acts in a natural way on $\Omega_{\bar{\Lambda}^{(N)}}, C_{\bar{\Lambda}^{(N)}}$ and on the space of states on $C_{\bar{\Lambda}^{(N)}}$. These actions are denoted by $\theta_{k}$ too. Note that $\theta_{k}$ is also well-defined for $k \in \mathbb{Z}+\frac{1}{2}$. The state $\bar{\mu}_{\text {alt }}^{(N)}$ is $\theta_{k}$-invariant for all $k \in \mathbb{Z}$, while the state $\bar{\mu}_{\text {inv }}^{(N)}$ is $\tau_{1}$-invariant. Note that if $\mu_{2}^{(N)}$ is $\theta_{1 / 2}$-invariant then $\bar{\mu}_{\text {alt }}^{(N)}=\bar{\mu}_{\text {inv }}^{(N)}$. If $\tau^{(a)} \mu^{(\infty)}=\mu_{2}^{(\infty)}$ for some $a \in \mathbb{Z}^{d}$ with $a_{1}=0$, then also $\tau^{(a)} \bar{\mu}_{\text {alt }}^{(\infty)}=\bar{\mu}_{\text {alt }}^{(\infty)}$ and $\tau^{(a)} \bar{\mu}_{\text {inv }}^{(\infty)}=\bar{\mu}_{\text {inv }}^{(\infty)}$. By construction the states $\bar{\mu}_{\text {alt }}^{(\infty)}$ and $\bar{\mu}_{\text {inv }}^{(\infty)}$ have the Markov property with respect to $P_{k}$ for all $k \in \mathbb{Z}$. [For instance considering $\bar{\mu}_{\text {alt }}^{(\infty)}$ one readily verifies that $\mathbb{E}_{\left(\mathbb{Z}^{d}\right)_{0,-}}^{\bar{\mu}_{\text {alt }}^{(\infty)}}\left(f_{0} f_{1} \cdots f_{n}\right)=f_{0} \mathbb{E}_{\downarrow}^{(\infty)}\left(\tilde{f}_{1}\left(\mathbb{E}_{\uparrow}^{(\infty)}\left(\tilde{f}_{2} \cdots \mathbb{E}_{(-1)^{n}}\right.\right.\right.$ $\left.\left.\left(\tilde{f}_{n}\right) \cdots\right)\right)$ ), where $f_{k} \in C_{\boldsymbol{P}_{k}}$ and $n \in \mathbb{N}$ and where the same definition of $\tilde{f}_{l}$ has been used as above for $l=1, \ldots, n$.] For $n \in \mathbb{N}$ and $\tilde{\mu}$ a state on $C_{\bar{\Lambda}^{(n)}}$ we denote by $\tilde{\mu}^{\otimes}$ the product state on $C(\Omega)$ defined by

$$
\tilde{\mu}^{\otimes}=\prod_{\left(n_{2}, \ldots, n_{d}\right) \in \mathbb{Z}^{d-1}}^{\otimes} \tau^{\left(\left(0,2 n n_{2}, \ldots, 2 n n_{d}\right)\right)} \tilde{\mu} .
$$

Consider the situation where $\mu_{2}^{(\infty)}$ is a state on $C_{P_{0} \cup P_{1}}$ and $\mu_{2}^{(n)}$ is, for $n \in \mathbb{N}$, defined as the state on $C_{P_{0}(n) \cup P_{1}(n)}$ obtained by restricting $\mu_{2}^{(\infty)}$, i.e.,

$$
\mu_{2}^{(n)}=\mu_{2}^{(\infty)} \uparrow_{C_{P_{0}(n) \cup P_{1}(n)}} .
$$

Like above define the states $\left\{\bar{\mu}_{\text {alt }}^{(n)}\right\}_{n \in \mathbb{N}}, \bar{\mu}_{\text {alt }}^{(\infty)}$ and, assuming $\tau_{1}\left(\mu_{2}^{(\infty)} \uparrow_{C_{P_{0}}}\right)=\mu_{2}^{(\infty)} \uparrow_{C_{P_{1}}}$, also the states $\left\{\bar{\mu}_{\text {inv }}^{(n)}\right\}_{n \in \mathbb{N}}$ and $\bar{\mu}_{\text {inv }}^{(\infty)}$.

Lemma 1. Let $n_{0} \in \mathbb{N}, n_{0} \geqq 1$. Let $H$ be the subgroup of $\mathbb{Z}^{d}$ generated by $\left\{a^{(i)}\right\}_{i=2, \ldots, d}$, where $a_{j}^{(i)}=n_{0} \delta_{i, j}$. Assume $\mu_{2}^{(\infty)}$ is H-invariant.

1) Define for $k \in \mathbb{N}, k \geqq 1$ the state $\rho_{\text {alt }}^{(k)}$ on $C(\Omega)$ by

Then

$$
\rho_{\mathrm{alt}}^{(k)}=\frac{1}{(2 k)^{d-1}} \sum_{a \in H \cap P_{0}\left(k n_{0}\right)} \tau^{(a)} \bar{\mu}_{\mathrm{alt}}^{\left(k n_{0}\right) \otimes} .
$$

$$
\underset{k \rightarrow \infty}{\text { weak }^{*} \lim } \rho_{\mathrm{alt}}^{(k)}=\bar{\mu}_{\mathrm{alt}}^{(\infty)}
$$


2) Assume $\tau_{1}\left(\mu_{2}^{(\infty)} \uparrow_{C_{P_{0}}}\right)=\mu_{2}^{(\infty)} \uparrow_{C_{P_{1}}}$. Define for $k \in \mathbb{N}, k \geqq 1$ the state $\rho_{\mathrm{inv}}^{(k)}$ on $C(\Omega)$ by

$$
\rho_{\mathrm{inv}}^{(k)}=\frac{1}{(2 k)^{d-1}} \sum_{a \in H \cap P_{0}\left(k n_{0}\right)} \tau^{(a)} \bar{\mu}_{\mathrm{inv}}^{\left(k n_{0}\right) \otimes} .
$$

Then

$$
\underset{k \rightarrow \infty}{\text { weak* }} \lim \rho_{\text {inv }}^{(k)}=\bar{\mu}_{\text {inv }}^{(\infty)} .
$$

Proof. The proof of 2) parallels the proof of 1). We therefore only present the proof of 1).

1) Take $f=f_{0} f_{1}, \ldots, f_{L}$ with $f_{l} \in C_{P_{l}(N)}$ for $l=0, \ldots, L$ and some $N<\infty$. It is sufficient to prove

$$
\lim _{k \rightarrow \infty} \rho_{\mathrm{alt}}^{(k)}(f)=\bar{\mu}_{\mathrm{alt}}^{(\infty)}(f)
$$

Choose $\varepsilon: 0<\varepsilon \in \mathbb{R}$.

Since the monotone sequences of projections $\left\{\mathbb{E}_{ \pm \uparrow}^{(n)}\right\}_{n \in \mathbb{N}}$ converge strongly to $\mathbb{E}_{ \pm \uparrow}^{(\infty)}$, there exists $\tilde{m} \in \mathbb{N}$ such that for $m \geqq \tilde{m}$,

$$
\left\|\left(\mathbb{E}_{(-1)^{l_{\uparrow}}(m)}^{(m)}-\mathbb{E}_{(-1)^{l_{\uparrow}}}^{(\infty)}\right)\left(\tilde{f}_{l} \mathbb{E}_{(-1)^{l+1} \uparrow}^{(\infty)}\left(\tilde{f}_{l+1} \cdots \tilde{f}_{L-1} \mathbb{E}_{(-1)^{L} \uparrow}^{(\infty)}\left(\tilde{f}_{L}\right) \cdots\right)\right)\right\|_{L^{2}} \leqq \varepsilon
$$

for all $l=2, \ldots, L$. Thus for $a \in H$ and $m \geqq \tilde{m}$,

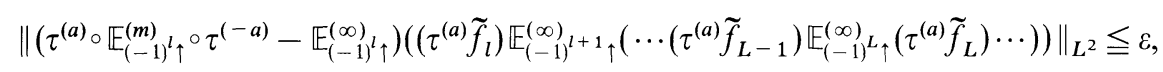

where we used $\mathbb{E}_{(-1)^{\prime} \uparrow}^{(\infty)} \tau^{(a)}=\tau^{(a)} \circ \mathbb{E}_{(-1)^{l} \uparrow}^{(\infty)}$ and the fact that $H$ acts isometrically on the $L^{2}$ space.

Both of these last properties are consequences of the $H$-invariance of $\mu_{2}^{(\infty)}$. If $\tau^{(a)} P_{0}(m) \subseteq P_{0}(n)$ then,

$$
\tau^{(a)} \circ \mathbb{E}_{(-1)^{l} \uparrow^{l(m)} \circ \tau^{(-a)} \leqq \mathbb{E}_{(-1)^{l} \uparrow}^{(n)}} .
$$

Hence, for $a \in \chi_{H}(\tilde{m} ; n)$ where $X_{H}(\tilde{m} ; n)=\left\{a \in H \mid \tau^{(a)} P_{0}(\tilde{m}) \subseteq P_{0}(n)\right\}$,

$$
\begin{aligned}
& \|\left(\tau^{(a)} f_{0}\right)\left(\tau^{(a)} f_{1}\right) \mathbb{E}_{\uparrow}^{(n)}\left(( \tau ^ { ( a ) } \tilde { f } _ { 2 } ) \mathbb { E } _ { \downarrow } ^ { ( n ) } \left(\left(\tau^{(a)} f_{3}\right) \cdots\left(\tau^{(a)} \tilde{f}_{l-1}\right)\left(\mathbb{E}_{(-1)^{l} \uparrow}^{(n)}-\mathbb{E}_{(-1)^{l^{l}}}^{(\infty)}\right)\right.\right. \\
& \left.\left.\left.\cdot\left(\left(\tau^{(a)} \tilde{f}_{l}\right) \mathbb{E}_{(-1)^{(+1}{ }_{\uparrow}(\infty)}\left(\cdots\left(\tau^{(a)} \tilde{f}_{L-1}\right) \mathbb{E}_{\left.(-1)^{L}\right)_{\uparrow}\left(\tau^{(a)}\right.}^{(a)} \tilde{f}_{L}\right) \cdots\right)\right) \cdots\right)\right)\left\|_{L^{2}} \leqq \varepsilon \prod_{k=0}^{l-1}\right\| f_{k} \|_{\infty}
\end{aligned}
$$

for $l=2, \ldots, d$. For $a \in X_{H}(\tilde{m} ; n)$ one thus readily deduces,

$$
\begin{aligned}
& \|\left(\tau^{(a)} f_{0}\right)\left(\tau^{(a)} f_{1}\right) \mathbb{E}_{\uparrow}^{(n)}\left(\left(\tau^{(a)} \tilde{f}_{2}\right) \mathbb{E}_{\downarrow}^{(n)}\left(\cdots \mathbb{E}_{(-1)^{L}}^{(n)}\left(\tau^{(a)} \tilde{f}_{L}\right) \cdots\right)\right) \\
& -\left(\tau^{(a)} f_{0}\right)\left(\tau^{(a)} f_{1}\right) \mathbb{E}_{\uparrow}^{(\infty)}\left(\left(\tau^{(a)} \tilde{f}_{2}\right) \mathbb{E}_{\downarrow}^{(\infty)}\left(\cdots \mathbb{E}_{(-1)^{L} \uparrow}^{(\infty)}\left(\tau^{(a)} \tilde{f}_{L}\right) \cdots\right)\right) \|_{L^{2}} \\
& \leqq\left\|f_{0}\right\|_{\infty}\left\|f_{1}\right\|_{\infty}\left[\varepsilon+\varepsilon \sum_{l=3}^{L} \prod_{k=2}^{l-1}\left\|f_{k}\right\|_{\infty}\right] \\
& \equiv \varepsilon C_{f_{0}, \ldots, f_{L}} .
\end{aligned}
$$

From the definitions of $\bar{\mu}_{\text {alt }}^{(n) \otimes}$ and $\bar{\mu}_{\text {alt }}^{(\infty)}$ it follows therefore

$$
\left|\bar{\mu}_{\mathrm{alt}}^{(n) \otimes}\left(\tau^{(a)} f\right)-\bar{\mu}_{\mathrm{alt}}^{(\infty)}\left(\tau^{(a)} f\right)\right| \leqq \varepsilon C_{f_{0}, \ldots, f_{L}}
$$


whenever $a \in X_{H}(\tilde{m} ; n)$ and $n \geqq N$. Consider the estimate

$$
\begin{aligned}
& \left|\rho_{\text {alt }}^{(k)}(f)-\bar{\mu}_{\text {alt }}^{(\infty)}(f)\right| \leqq \frac{1}{(2 k)^{d-1}} \sum_{-a \in H \cap P_{0}\left(k n_{0}\right) \cap X_{H}\left(\tilde{m}, k n_{0}\right)^{c}}\left|\left(\tau^{(a)} \bar{\mu}_{\text {alt }}^{(n) \otimes}\right)(f)-\bar{\mu}_{\text {alt }}^{(\infty)}(f)\right|
\end{aligned}
$$

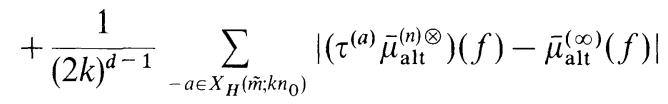

$$
\begin{aligned}
& \leqq \frac{1}{(2 k)^{d-1}} \sum_{-a \in H \cap P_{0}\left(k n_{0}\right) \cap X_{\left.H^{(m)} ; n_{0}\right)^{c}}} 2\|f\|_{\infty} \\
& +\frac{1}{(2 k)^{d-1}} \sum_{-a \in X_{H}\left(\tilde{m} ; k n_{0}\right)} \varepsilon C_{f_{0}, \ldots, f_{L}} .
\end{aligned}
$$

The first sum in the last upper bound is a sum over a number of terms bounded by a constant times $(2 k)^{d-2}$.

Hence,

$$
\underset{k \rightarrow \infty}{\limsup }\left|\rho_{\mathrm{alt}}^{(k)}(f)-\bar{\mu}_{\mathrm{alt}}^{(\infty)}(f)\right| \leqq \varepsilon C_{f_{0}, \ldots, f_{L}},
$$

and the lemma follows.

For $m, L, K \in \mathbb{N}$ define $\Lambda(m ; L, K) \subset \mathbb{Z}^{d}$ as

$$
\Lambda(m ; L, K)=\left\{x \in \mathbb{Z}^{d} \mid-L \leqq x_{1} \leqq K+1 \quad \text { and } \quad-m \leqq x_{i}<m \text { for } i=2, \ldots, d\right\} .
$$

Let $M_{+1}$ be the Radon-Nikodym derivative of the restriction of $\bar{\mu}_{\text {alt }}^{(n)}(=$ the restriction of $\mu$ ) to $C_{\Lambda_{2}^{(n)}}$ with respect to $\mu_{0}^{\left(\Lambda_{2}^{(n)}\right)}$. Define $M_{-1}=\theta_{1 / 2} M_{+1}$. Furthermore define

$$
\underline{M}_{+1}=\int_{\Omega_{P_{1}(n)}} d \mu_{0}^{\left(P_{1}(n)\right)} M_{+1}
$$

and

$$
\underline{M}_{-1}=\int_{\Omega_{P_{1}(n)}} d \mu_{0}^{\left(P_{1}(n)\right)} M_{-1}
$$

Then the Radon-Nikodym derivative, $\bar{\mu}^{(m)^{\left(\Lambda^{(m, L, K)}\right)}}$ of the restriction of $\bar{\mu}_{\text {alt }}^{(n)}$ to $C_{\Lambda^{(m, L, K)}}$ with respect to $\mu_{0}^{\left(\Lambda^{(m, L, K)}\right)}$ is given by

$$
\bar{\mu}_{\mathrm{alt}}^{(n)(\Lambda(m: L, K))}=\frac{\tau_{1}^{-L} \underline{M}_{(-1)^{-L}}}{\tau_{1}^{-L+1} \underline{M}_{(-1)}{ }^{-L+1}} \cdots \frac{\tau_{1}^{-1} M_{-1}}{\underline{M}_{+1}} \cdot M_{+1} \cdot \frac{\tau_{1} M_{-1}}{\tau_{1} \underline{M}_{-1}} \cdots \frac{\tau_{1}^{K} M_{(-1)^{M}}}{\tau_{1}^{K} \underline{M}_{(-1)^{K}}} .
$$

(Note that $M_{+1}, M_{-1}, \underline{M}_{+1}$, and $\underline{M}_{-1}$ can be assumed to be continuous functions in case $\mu_{2}^{(\infty)}$ is the restriction of a Gibbs state of $\Lambda_{2}^{(\infty)}$.) Then

$$
S_{\Lambda(m ; L, L)}\left(\bar{\mu}_{\mathrm{alt}}^{(m)}\right)=(2 L+1) S_{P_{0}(m)} \cup P_{1}(m)\left(\mu_{2}^{(\infty)}\right)-L S_{P_{0}(m)}\left(\mu_{2}^{(\infty)}\right)-L S_{P_{1}(m)}\left(\mu_{2}^{(\infty)}\right) .
$$

Reasoning similarly one also obtains

$$
S_{\Lambda(m ; L, L)}\left(\bar{\mu}_{\text {inv }}^{(m)}\right)=(2 L+1) S_{P_{0}(m) \cup P_{1}(m)}\left(\mu_{2}^{(\infty)}\right)-2 L S_{P_{0}(m)}\left(\mu_{2}^{(\infty)}\right) .
$$

\section{Lemma 2}

a) Consider the situation in 1) of Lemma 1. Then 


$$
s\left(\bar{\mu}_{\mathrm{alt}}^{(\infty)}\right)=\lim _{n \rightarrow \infty} \frac{1}{(2 n)^{d-1}}\left[S_{P_{0}(n) \cup P_{1}(n)}\left(\mu_{2}^{(\infty)}\right)-\frac{1}{2} S_{P_{0}(n)}\left(\mu_{2}^{(\infty)}-\frac{1}{2} S_{P_{1}(n)}\left(\mu_{2}^{(\infty)}\right)\right] .\right.
$$

b) Consider the situation in 2) of Lemma 1. Then

$$
s\left(\bar{\mu}_{\mathrm{inv}}^{(\infty)}\right)=\lim _{n \rightarrow \infty} \frac{1}{(2 n)^{d-1}}\left[S_{P_{0}(n) \cup P_{1}(n)}\left(\mu_{2}^{(\infty)}\right)-S_{P_{0}(n)}\left(\mu_{2}^{(\infty)}\right)\right] .
$$

Proof. The proof of $b$ ) is analogous to the proof of $a$ ).

We present the proof of a).

a). By Lemma 1 one obtains, using upper-semicontinuity of the entropy,

$$
s\left(\bar{\mu}_{\text {alt }}^{(\infty)}\right) \geqq \limsup _{k \rightarrow \infty} s\left(\rho_{\text {alt }}^{(k)}\right)=\underset{k \rightarrow \infty}{\limsup } s\left(\bar{\mu}_{\text {alt }}^{\left(k n_{0}\right)}\right) .
$$

From $(*)$ for $n \in \mathbb{N}, n \geqq 1$,

$$
s\left(\bar{\mu}_{\mathrm{alt}}^{(n)}\right)=\frac{1}{(2 n)^{d-1}}\left[S_{P_{0}(n) \cup P_{1}(n)}\left(\mu_{2}^{(\infty)}\right)-\frac{1}{2} S_{P_{0}(n)}\left(\mu_{2}^{(\infty)}\right)-\frac{1}{2} S_{P_{1}(n)}\left(\mu_{2}^{(\infty)}\right)\right] .
$$

Therefore

$$
\begin{aligned}
s\left(\bar{\mu}_{\mathrm{alt}}^{(\infty)}\right) & \geqq \limsup _{k \rightarrow \infty} \frac{1}{\left(2 k n_{0}\right)^{d-1}}\left[S_{P_{0}\left(k n_{0}\right) \cup P_{1}\left(k n_{0}\right)}\left(\mu_{2}^{(\infty)}\right)-\frac{1}{2} S_{P_{0}\left(k n_{0}\right)}\left(\mu_{2}^{(\infty)}\right)-\frac{1}{2} S_{P_{1}\left(k n_{0}\right)}\left(\mu_{2}^{(\infty)}\right)\right] \\
& =\lim _{n \rightarrow \infty} \frac{1}{(2 n)^{d-1}}\left[S_{P_{0}(n) \cup P_{1}(n)}\left(\mu_{2}^{(\infty)}\right)-\frac{1}{2} S_{P_{0}(n)}\left(\mu_{2}^{(\infty)}\right)-\frac{1}{2} S_{P_{1}(n)}\left(\mu_{2}^{(\infty)}\right)\right] .
\end{aligned}
$$

On the other hand, by repeated use of the strong subadditivity property of the entropy,

$$
\begin{aligned}
S_{\Lambda\left(l n_{0} ; l l\right)}\left(\bar{\mu}_{\mathrm{alt}}^{(\infty)}\right) \leqq & S_{\Lambda\left(l n_{0}: l l,-1\right)}\left(\bar{\mu}_{\mathrm{alt}}^{(\infty)}\right)+S_{P_{l}\left(l n_{0}\right) \cup P_{l-1}\left(l n_{0}\right)}\left(\bar{\mu}_{\mathrm{alt}}^{(\infty)}\right)-S_{P_{l}\left(l n_{0}\right)}\left(\bar{\mu}_{\mathrm{alt}}^{(\infty)}\right) \\
\leqq & S_{\Lambda\left(l n_{0} ; l, l-2\right)}\left(\bar{\mu}_{\mathrm{alt}}^{(\infty))}\right)+S_{P_{l-1}\left(l n_{0}\right) \cup P_{l-2}\left(l n_{0}\right)}\left(\bar{\mu}_{\mathrm{alt}}^{(\infty))}\right) \\
& \quad-S_{P_{l-1}\left(l n_{0}\right)}\left(\bar{\mu}_{\mathrm{alt}}^{(\infty)}\right)+S_{P_{l}\left(l n_{0}\right) \cup P_{l-1}\left(l n_{0}\right)}\left(\bar{\mu}_{\mathrm{alt}}^{(\infty)}\right)-S_{P_{l}\left(l n_{0}\right)}\left(\bar{\mu}_{\mathrm{alt}}^{(\infty)}\right) \\
\leqq & \cdots \cdots \cdots \\
\leqq & \cdots \cdots \cdots \\
& \quad \vdots \\
\leqq & (2 l+1) S_{P_{0}\left(l n_{0}\right) \cup P_{1}\left(l n_{0}\right)}\left(\bar{\mu}_{\mathrm{alt}}^{(\infty)}\right)-l S_{P_{0}\left(l n_{0}\right)}\left(\bar{\mu}_{\mathrm{alt}}^{(\infty)}\right)-l S_{P_{1}\left(l n_{0}\right)}\left(\bar{\mu}_{\mathrm{alt}}^{(\infty)}\right)
\end{aligned}
$$

We used that by $\theta_{k}$-invariance of $\bar{\mu}_{\mathrm{alt}}^{(\infty)}$ for all $k \in \mathbb{Z}$,

$$
S_{P_{k}\left(\left(n_{0}\right) \cup P_{k+1}\left(l n_{0}\right)\right.}\left(\bar{\mu}_{\mathrm{alt}}^{(\infty)}\right)=S_{P_{0}\left(l n_{0}\right) \cup P_{1}\left(l_{0}\right)}\left(\bar{\mu}_{\mathrm{alt}}^{(\infty)}\right)
$$

and

$$
S_{P_{2 k}\left(l_{0}\right)}\left(\bar{\mu}_{\mathrm{alt}}^{(\infty)}\right)=S_{P_{0}\left(l_{0}\right)}\left(\bar{\mu}_{\mathrm{alt}}^{(\infty)}\right)
$$

and

$$
S_{P_{2 k+1}\left(l n_{0}\right)}\left(\bar{\mu}_{\mathrm{alt}}^{(\infty)}\right)=S_{P_{1}\left(l_{0}\right)}\left(\bar{\mu}_{\mathrm{alt}}^{(\infty)}\right)
$$


Therefore,

$$
\begin{aligned}
& s\left(\bar{\mu}_{\mathrm{alt}}^{(\infty)}\right)=\lim _{l \rightarrow \infty}\left[\frac{1}{2(l+1)\left(2 \ln _{0}\right)^{d-1}} S_{\Lambda\left(n_{0}, l, l\right)}\left(\bar{\mu}_{\mathrm{alt}}^{(\infty)}\right)\right] \\
& \leqq \lim _{l \rightarrow \infty} \frac{1}{\left(2 \ln _{0}\right)^{d-1}}\left[S_{P_{0}\left(\left(n_{0}\right) \cup P_{1}\left(l n_{0}\right)\right.}\left(\bar{\mu}_{\text {alt }}^{(\infty)}\right)-\frac{1}{2} S_{P_{0}\left(n_{0}\right)}\left(\bar{\mu}_{\text {alt }}^{(\infty)}\right)-\frac{1}{2} S_{P_{1}\left(l n_{0}\right)}\left(\bar{\mu}_{\text {alt }}^{(\infty)}\right)\right] \\
& =\lim _{n \rightarrow \infty} \frac{1}{(2 n)^{d-1}}\left[S_{P_{0}(n) \cup P_{1}(n)}\left(\mu_{2}^{(\infty)}\right)-\frac{1}{2} S_{P_{0}(n)}\left(\mu_{2}^{(\infty)}\right)-\frac{1}{2} S_{P_{1}(n)}\left(\mu_{2}^{(\infty)}\right)\right] .
\end{aligned}
$$

Consider now an interaction $\Phi$ like in the introductory section, i.e., $\Phi$ is nonzero only round faces of $\mathbb{Z}^{d}$ and invariant under a group $G$ with finite index in $\mathbb{Z}^{d}$.

Theorem 1. Let $\mu$ be a G-invariant Gibbs state for the interaction $\Phi$, where $\left|\mathbb{Z}^{d} / G\right|<\infty$. Put

$$
\mu_{2}^{(\infty)}=\mu \uparrow_{C_{P_{0} \cup P_{1}}} .
$$

1) Assume that $\Phi$ and $\mu$ are $\theta_{k}$-invariant for all $k \in \mathbb{Z}$. Construct the state $\bar{\mu}_{\text {alt }}^{(\infty)}$ from $\mu_{2}^{\infty}$. Then $\bar{\mu}_{\text {alt }}^{(\infty)}$ is a Gibbs state for $\Phi$.

2) Assume that $\Phi$ and $\mu$ are invariant for translation over $(k, 0, \ldots, 0) \in \mathbb{Z}^{d}$ for all $k \in \mathbb{Z}$. Construct $\bar{\mu}_{\mathrm{inv}}^{(\infty)}$ from $\mu_{2}^{(\infty)}$. Then $\bar{\mu}_{\mathrm{inv}}^{(\infty)}$ is a Gibbs state for $\Phi$.

Proof. Since $G$ has finite index in $\mathbb{Z}^{d}$ there exists $n_{0} \geqq 1, n_{0} \in \mathbb{N}$ such that $\left\{a^{(i)}\right\}_{i=2, \ldots, d} \subset G$, where $a_{j}^{(i)}=n_{0} \delta_{i, j}$. Let $H$ be the subgroup of $G$ generated by $\left\{a^{(i)}\right\}_{i=2, \ldots, d}$.

1) By the same reasoning as in the last part of the proof of Lemma 2,

$$
s(\mu) \leqq \lim _{n \rightarrow \infty} \frac{1}{(2 n)^{d-1}}\left[S_{P_{0}(n) \cup P_{1}(n)}\left(\mu_{2}^{(\infty)}\right)-\frac{1}{2} S_{P_{0}(n)}\left(\mu_{2}^{(\infty)}\right)-\frac{1}{2} S_{P_{1}(n)}\left(\mu_{2}^{(\infty)}\right)\right] .
$$

Thus $s(\mu) \leqq s\left(\mu_{\text {alt }}^{(\infty)}\right)$. Furthermore, for pairs $(x, X)$ with $x \in P_{0} \cup P_{1}$ and $X \subset \mathbb{Z}^{d}$ such that $x \in X$ is the first element of $X$ (in the lexicographic order of $\mathbb{Z}^{d}$ ) and $\Phi \circ \alpha_{X} \neq 0$, we define $\hat{X}(x, X) \subset \mathbb{Z}^{d}$ by

a) if $X \subset P_{0} \cup P_{1}$, then $\hat{X}(x, X)=X$,

b) if $X \not P_{0} \cup P_{1}$, then $\hat{X}(x, X)=\theta_{1} X$.

Thus always $\hat{X}(x, X) \subset P_{0} \cup P_{1}$. Then, since the states $\mu$ and $\bar{\mu}_{\text {alt }}^{(\infty)}$ as well as the potential $\Phi$ are invariant under the reflection $\theta_{1}$,

$$
\mu\left(A_{\Phi}^{(x)}\right)=\mu\left(\sum_{X}^{(x)} \Phi \circ \alpha_{\hat{X}(x, X)}\right)
$$

and

$$
\bar{\mu}_{\mathrm{alt}}^{(\infty)}\left(A_{\Phi}^{(x)}\right)=\bar{\mu}_{\mathrm{alt}}^{(\infty)}\left(\sum_{X}^{(x)} \Phi \circ \alpha_{\hat{X}(x, X)}\right)
$$

for $x \in P_{0} \cup P_{1}$. But

$$
\mu\left(\Phi \circ \alpha_{\hat{X}(x, X)}\right)=\bar{\mu}_{\text {alt }}^{(\infty)}\left(\Phi \circ \alpha_{\hat{X}(x, X)}\right)
$$


for $x \in P_{0} \cup P_{1}$. Therefore, if $x \in P_{0} \cup P_{1}$,

$$
\mu\left(A_{\Phi}^{(x)}\right)=\bar{\mu}_{\mathrm{alt}}^{(\infty)}\left(A_{\Phi}^{(x)}\right) .
$$

Next note that $\mu$ and $\bar{\mu}_{\text {alt }}^{(\infty)}$ as well as $\Phi$ are invariant under the group generated by $H$ and $(2,0, \ldots, 0) \in \mathbb{Z}^{d}$.

An application of Theorem 0 for this last group (with $M_{0}=P_{0}\left(n_{0}\right) \cup P_{1}\left(n_{0}\right)$ ) gives, from the inequality

$$
s\left(\bar{\mu}_{\mathrm{alt}}^{(\infty)}\right)-\frac{1}{\left(2 n_{0}\right)^{d-1}} \sum_{x \in P_{0}\left(n_{0}\right) \cup P_{1}\left(n_{0}\right)} \bar{\mu}_{\mathrm{alt}}^{(\infty)}\left(A_{\Phi}^{(x)}\right) \geqq s(\mu)-\frac{1}{\left(2 n_{0}\right)^{d-1}} \sum_{x \in P_{0}\left(n_{0}\right) \cup P_{1}\left(n_{0}\right)} \mu\left(A_{\Phi}^{(x)}\right),
$$

that $s\left(\bar{\mu}_{\mathrm{alt}}^{(\infty)}\right)=s(\mu)$ and that $\bar{\mu}_{\mathrm{alt}}^{(\infty)}$ is a Gibbs state for $\Phi$.

2) The proof of 2) is analogous to the proof of 1 ).

\section{Corollary 1.}

1) Consider the situation described in Theorem 1.1). Let $G_{0}^{(2)}$ be the group generated by $G \cap P_{0}$ and $(2,0, \ldots, 0) \in \mathbb{Z}^{d}$. Assume $\Phi$ is $\theta_{k}$-invariant for all $k \in \mathbb{Z}$. Consider the condition $C_{1}$ :

$C_{1}\left\{\begin{array}{l}\text { If the Gibbs states } \mu_{1} \text { and } \mu_{2} \text { for the potential } \Phi \text { are both } G_{0}^{(2)} \text {-and } \\ \theta_{k} \text {-invariant for all } k \in \mathbb{Z} \text { and } \mu_{1} \uparrow_{C_{P_{0} \cup P_{1}}}=\mu_{2} \uparrow_{C_{P_{0} \cup P_{1}}} \text {, then } \mu_{1}=\mu_{2} .\end{array}\right.$ If $C_{1}$ holds then every $G_{0}^{(2)}$ - and $\theta_{k}$-invariant (for all $k \in \mathbb{Z}$ ) Gibbs state for the potential $\Phi$ has the Markov property with respect to $P_{k}$ for all $k \in \mathbb{Z}$.

2) Consider the situation described in Theorem 1.2). Assume $(1,0, \ldots, 0) \in G$. Consider the condition $\mathrm{C}_{2}$ :

$$
C_{2}\left\{\begin{array}{l}
\text { If the Gibbs states } \mu_{1} \text { and } \mu_{2} \text { for the potential } \Phi \text { are both } G \text {-invariant and } \\
\mu_{1} \uparrow_{C_{P_{0} \cup P_{1}}}=\mu_{2} \uparrow_{C_{P_{0} \cup P_{1}}} \text {, then } \mu_{1}=\mu_{2} .
\end{array}\right.
$$

If $\mathrm{C}_{2}$ holds then every G-invariant Gibbs state has the Markov property with respect to $P_{k}$ for all $k \in \mathbb{Z}$.

Note that Corollary 1 implies the following. If the states determining equilibrium are recognizable by observations on a plaquette (and have some invariances), Theorem 1 implies that Markov properties hold with respect to $P_{k}$ for all $k \in \mathbb{Z}$. A particular case of such situations occurs for models with a phase diagram described by the expectation value of the spin on a lattice site (magnetization).

Note. In a forthcoming paper we show that for models with nearest-neighbor interactions (and with some periodicity properties) leading to an invertible transfer matrix (for instance the $q$-state Potts model for nontrivial coupling) the conditions stated in Corollary 1 are met.

In two dimensions a further relation between the states $\mu$ and $\bar{\mu}_{\mathrm{alt}}^{(\infty)}$ (or $\mu$ and $\left.\bar{\mu}_{\text {inv }}^{(\infty)}\right)$ can be given which shows that these states do not become disjoint over finite distances in the 1-direction. We will make use of the concept of relative entropy of states. When $\mu$ and $v$ are probability measures on $\Omega$ the relative entropy $S(\mu \mid v)$ is defined by

$$
S(\mu \mid v)=-\int \frac{d \mu}{d v} \ln \left(\frac{d \mu}{d v}\right) d v
$$


if $\mu \ll v$ and the Radon-Nikodym derivative of $\mu$ with respect to $v,(d \mu / d v)$, is such that $(d \mu / d v) \ln (d \mu / d v)$ is $v$-integrable. Otherwise $S(\mu \mid v)=-\infty$ by definition. If $\left\{v_{n}\right\}_{n \in \mathbb{N}}$ is a sequence of states converging to the weak* topology to a state $v$ and $\mu$ is a state such that $S\left(\mu \mid v_{n}\right) \geqq-k$ for all $n \in \mathbb{N}$, where $k \geqq 0$ is some finite constant, then $\mu \ll v$. Application of this result together with some more details can be found in [7].

Theorem 2. Let the lattice be two dimensional, i.e., $\mathbb{Z}^{2}$. We use notation introduced in the proof of Lemma 2.

1) Consider the situation described in Theorem 1.1). Then

$$
\mu \uparrow_{C_{\Lambda(\infty ; L, L)}} \ll \bar{\mu}_{\mathrm{alt}}^{(\infty)} \uparrow_{C_{\Lambda(\infty ; L, L)}}
$$

for all $L \in \mathbb{N}$.

2) Consider the situation described in Theorem 1.2). Then

$$
\mu \uparrow_{C_{\Lambda(\infty ; L, L)}} \ll \bar{\mu}_{\mathrm{inv}}^{(\infty)} \uparrow_{C_{\Lambda(\infty: L, L)}}
$$

for all $L \in \mathbb{N}$.

Proof. We only present the proof of 1) since the proof 2) is analogous. 1) introduce $n_{0}: 1 \leqq n_{0} \in \mathbb{N}$ like in the proof of Theorem 1 . Let $\mathbb{E}_{k}: L^{2}\left(\mu ; \mathfrak{B}_{\Lambda(\infty ; L, L)}\right) \rightarrow L^{2}\left(\mu ; \mathfrak{B}_{\Lambda\left(k n_{0}, L, L\right)}\right)$ be the orthogonal projection for $k \in \mathbb{N}$.

Note that the restrictions of two Gibbs states to a finite volume are mutually absolutely continuous. Thus the following definition of the state $\tilde{\mu}_{k}$ on $C_{\Lambda(\infty ; L, L)}$ is justified:

$$
\tilde{\mu}_{k}(f)=\int d \bar{\mu}_{\mathrm{alt}}^{\left(k n_{0}\right)} \mathbb{E}_{k}(f)
$$

for $f \in C_{A(x ; L, L)}$. The Radon-Nikodym derivative $h_{k}$ of $\mu \uparrow_{C_{A(\infty, L, L)}}$ with respect to $\tilde{\mu}_{k}$ is given by

$$
h_{k}=\frac{\mu^{\left(\Lambda\left(k n_{0}, L, L\right)\right)}}{\bar{\mu}_{\mathrm{alt}}^{(k n)^{\left(\Lambda\left(k n_{0}, L, L\right)\right)}}} .
$$

Therefore

$$
\begin{aligned}
S\left(\mu \Gamma_{C_{A(\infty, L, L)}} \mid \tilde{\mu}_{k}\right) & =-\int d \tilde{\mu}_{k} h_{k} \ln h_{k}=-\int d \mu \ln h_{k} \\
& =-\int d \mu\left[\ln \left(\mu^{\left(\Lambda\left(k n_{0}, L, L\right)\right)}\right)-\ln \left(\bar{\mu}_{\mathrm{alt}}^{\left(k n_{0}\right)\left(\Lambda\left(k n_{0}, L, L\right)\right)}\right)\right] .
\end{aligned}
$$

From the product-type structure of the density of $\bar{\mu}_{\text {alt }}^{\left(k n_{0}\right)} \uparrow_{\left.\mathcal{B}_{A\left(k n_{0}, L\right.} L\right)}$ with respect to $\mu_{0}^{\left(\Lambda\left(k n_{0} ; L, L\right)\right)}$, like already used in the proof of Lemma 2 for calculating entropies, and the fact that $\mu$ coincides with $\bar{\mu}_{\mathrm{alt}}^{(k n)}$ on $C_{P_{l}\left(k n_{0}\right) \cup P_{l+1}\left(k n_{0}\right)}$, one obtains

$$
\int d \mu \ln \left(\bar{\mu}_{\mathrm{alt}}^{\left(k n_{0}\right)^{\left(A\left(k n_{0}, L, L\right)\right)}}\right)=\int d \mu_{\mathrm{alt}}^{\left(k n_{0}\right)} \ln \left(\bar{\mu}_{\mathrm{alt}}^{\left(k n_{0}\right)^{\left(\Lambda\left(k n_{0}, L, L\right)\right)}}\right) .
$$

Hence,

$$
\begin{aligned}
S\left(\left.\mu\right|_{C_{\Lambda(\infty ; L, L)}} \mid \tilde{\mu}_{k}\right) & =S_{\Lambda\left(k n_{0}, L, L\right)}(\mu)-S_{\Lambda\left(k n_{0}, L, L\right)}\left(\bar{\mu}_{\mathrm{alt}}^{\left(k n_{0}\right)}\right) \\
& =4 k n_{0} \sum_{l=0}^{L-2}\left[b_{l}^{\left(k n_{0}\right)}(\mu)-b_{l}^{\left(k n_{0}\right)}\left(\bar{\mu}_{\mathrm{alt}}^{\left(k n_{0}\right)}\right)\right]
\end{aligned}
$$


where

$$
b_{l}^{\left(k n_{0}\right)}(\cdot)=\frac{1}{4 k n_{0}}\left[S_{\Lambda\left(k n_{0}, L, L-2 l\right)}(\cdot)-S_{\Lambda\left(k n_{0} ; L, L-2(l+1)\right)}(\cdot)\right] .
$$

On the other hand

$$
s\left(\bar{\mu}_{\mathrm{alt}}^{\left(k n_{0}\right) \otimes}\right)=\frac{1}{2 k n_{0}}\left[S_{P_{0}\left(k n_{0}\right) \cup P_{1}\left(k n_{0}\right)}(\mu)-\frac{1}{2} S_{P_{0}\left(k n_{0}\right)}(\mu)-\frac{1}{2} S_{P_{1}\left(k n_{0}\right)}(\mu)\right]=b_{l}^{\left(k n_{0}\right)}\left(\bar{\mu}_{\mathrm{alt}}^{\left(k n_{0}\right)}\right)
$$

for $l=0, \ldots, L-2$.

The variational principle implies

$$
b_{l}^{\left(k n_{0}\right)}\left(\bar{\mu}_{\mathrm{alt}}^{\left(k n_{0}\right)}-\frac{1}{4 k n_{0}} \sum_{x \in P_{0}\left(k n_{0}\right) \cup P_{1}\left(k n_{0}\right)} \bar{\mu}_{\mathrm{alt}}^{\left(k n_{0}\right) \otimes}\left(A_{\Phi}^{(x)}\right) \leqq s(\mu)-\frac{1}{4 k n_{0}} \sum_{x \in P_{0}\left(k n_{0}\right) \cup P_{1}\left(k n_{0}\right)} \mu\left(A_{\Phi}^{(x)}\right) .\right.
$$

Since the number of $x \in P_{0}\left(k n_{0}\right) \cup P_{1}\left(k n_{0}\right)$, such that there exists $X \subset \mathbb{Z}^{d}$ with $\Phi$. $\alpha_{X} \neq 0, X$ has $x$ as first element in the lexicographic order of $\mathbb{Z}^{d}$, and $X \not \Lambda^{\left(k n_{0}\right)}$, is uniformly bounded

$$
s(\mu)-b_{l}^{\left(k n_{0}\right)}\left(\bar{\mu}_{\mathrm{alt}}^{\left(k n_{0}\right)}\right) \geqq \frac{-K}{4 k n_{0}},
$$

where $K \geqq 0$ is a constant independent of $k$. Thus

$$
S\left(\mu \uparrow_{C_{\Lambda(\infty ; L, L)}} \mid \tilde{\mu}_{k}\right) \geqq 4 k n_{0} \sum_{l=0}^{L-2}\left[b_{l}^{\left(k n_{0}\right)}(\mu)-s(\mu)\right]-K .
$$

Moreover

$$
b_{l}^{\left(k n_{0}\right)}(\mu) \geqq s(\mu) .
$$

One obtains

$$
S\left(\mu \uparrow_{C_{\Lambda(\infty, I, L)}} \mid \tilde{\mu}_{k}\right) \geqq-K .
$$

Theorem 2 1) therefore follows.

Acknowledgements. The author would like to thank A. G. Schlijper and M. Winnink for several stimulating discussions.

\section{References}

1. Goldstein, S.: Remarks on the global Markov property. Commun. Math. Phys. 74, 223-234 (1980)

2. Simon, B.: The $P(\Phi)_{2}$ Euclidean (Quantum) field theory. Princeton, New Jersey: Princeton University Press 1974

3. Föllmer, H.: On the global Markov property. In: Quantum fields-, algebras, processes. Streit, L., (ed.) pp. 293-302. Wien-New York: Springer 1980

4. Bellisard, J., Hфegh-Krohn, R.: Compactness and the maximal Gibbs state for random Gibbs fields on a lattice. Commun. Math. Phys. 84, 297-327 (1982)

5. Albeverio, S., Hфegh-Krohn, R.: The global Markov property for Euclidean and lattice fields. Phys. Lett. 84B, 89-90 (1979)

6. Schlijper, A. G.: Exact variational methods and cluster-variation approximations. J. Stat. Phys. 35, 285-301 (1984); and Variational Approximation in Classical Lattice Systems. Thesis, University of Groningen (1985) 
7. Fröhlich, J., Pfister, C.-E.: On the absence of spontaneous symmetry breaking and of crystalline ordering in two-dimensional systems. Commun. Math. Phys. 81, 277-298 (1982)

8. Ruelle, D.: Thermodynamic formalism. Reading, MA.: Addison Wesley 1978

9. Simon, B.: A remark on Dobrushin's uniqueness theorem. Commun. Math. Phys. 68, 183-185 (1979)

10. Israel, R. B.: Some examples concerning the global Markov property. Commun. Math. Phys. 105, $669-673(1986)$

Communicated by M. Aizenman

Received December 16, 1986; in revised form August 16, 1987 
